\title{
A Continued Fraction Expansion, with a Truncation Error Estimate, for Dawson's Integral
}

\author{
By J. H. McCabe
}

\begin{abstract}
A continued fraction expansion for Dawson's integral is considered and an estimate of the truncation errors of the convergents of this continued fraction is provided. The continued fraction is shown to provide a sequence of rational approximations to the integral which have good convergence for both small and large values of the argument.
\end{abstract}

\section{Introduction. Dawson's integral}

$$
F(x)=e^{-x^{2}} \int_{0}^{x} e^{t^{2}} d t
$$

is of importance in several physical problems. It occurs in such applications as heat conduction and spectroscopy, and in the theory of electrical oscillations in certain special vacuum tubes. The integral is closely related to the complex error function $w(z)$

$$
w(z)=e^{-z^{2}} \operatorname{erfc}(-i z)=e^{-z^{2}}+\frac{2 i}{\sqrt{\pi}} F(z)
$$

where erfc $\zeta$, the complementary error function, is given by

$$
\operatorname{erfc} \zeta=\frac{2}{\sqrt{\pi}} \int_{\zeta}^{\infty} e^{-t^{2}} d t
$$

The function $F(x)$ was first tabulated by Dawson [2] and since then extensive tabulations have appeared. Lohmander and Rittsten [6] give values to ten decimal places for a large range of values of $x$. Hummer [4] expands $F(x)$ as a series of Chebyshev polynomials, giving the coefficients to eighteen decimal places, while Cody et al. [1] provide a set of nearly best rational approximations to $F(x)$ for all real $x$, with relative accuracies up to twenty-two significant figures.

2. Series and Continued Fraction Expansions. Differentiating $F(z)$, we find that for all complex values of $z$ we obtain

$$
F^{\prime}(z)+2 z F(z)=1
$$

and further, for $k \geqq 1$, the derivatives of $F(z)$ can be shown to satisfy

$$
F^{(k+1)}(z)+2 z F^{(k)}(z)+2 k F^{(k-1)}(z)=0 .
$$

Received September 10, 1973.

AMS (MOS) subject classifications (1970). Primary 41A20, 30A82.

Copyright $\odot$ 1974. American Mathematical Society 
Thus if we know the value of $F(z)$ at any point we can evaluate the Taylor series of $F(z)$ about that point. In particular, since $F(0)=0$, we readily obtain the Maclaurin series

$$
F(z)=\sum_{k=0}^{\infty} \frac{(-)^{k} k ! 2^{2 k} z^{2 k+1}}{(2 k+1) !}
$$

The series (2.3) converges for all finite $z$. However, as Cody et al. [1] point out, practical convergence is delayed until $k$ becomes greater than $\left|z^{2}\right|-\frac{3}{2}$.

Corresponding to the series $(2.3)$ is the continued fraction expansion

$$
F(z)=\frac{z}{1}+\frac{2 z^{2}}{3}-\frac{4 z^{2}}{5}+\frac{6 z^{2}}{7}-\frac{8 z^{2}}{9}+\cdots .
$$

Naturally this continued fraction also converges throughout the finite complex plane and as Thacher [12] points out, it is uniformly more efficient than the series (2.3) and is numerically stable in the first octant of the complex plane. For large values of $|z|$ the convergence of (2.4), though faster than that of (2.3), is still slow.

When $|\arg z|<\pi / 4$ approximations to $F(z)$ for large values of $|z|$ can be obtained from the asymptotic series

$$
F(z) \sim \frac{1}{2 z}+\frac{1}{2^{2} z^{3}}+\frac{1}{2^{3} z^{5}}+\frac{1}{2^{4} z^{7}}+\cdots
$$

This series is divergent for all real values of $z$ but, provided it is truncated after a suitable number of terms, can supply good approximations to Dawson's integral: for $x=10$ an accuracy of twenty-five decimal places is obtained from twenty-four terms.

Corresponding to the series $(2.5)$ is the continued fraction

$$
F(z) \sim \frac{1}{2 z}-\frac{2}{2 z}-\frac{4}{2 z}-\frac{6}{2 z}-\cdots,
$$

which, like the series (2.5), is divergent for all real $z$ but once again can be used to provide approximations to Dawson's integral for large values of the argument. In such instances it is to be preferred to the continued fraction (2.4), even though the latter is convergent.

The two continued fractions given above each correspond to one series only. A third continued fraction expansion of $F(z)$ is

$$
F(z)=\frac{z}{1+2 z^{2}}-\frac{4 z^{2}}{3+2 z^{2}}-\frac{8 z^{2}}{5+2 z^{2}}-\frac{12 z^{2}}{7+2 z^{2}}-\cdots,
$$

and this expansion corresponds to the series (2.3) and to the series (2.5) simultaneously. The continued fraction belongs to a class of corresponding fractions, called $M$ fractions, which are constructed to correspond to a power series expansion about the origin and to another about the point at infinity simultaneously. This class of corresponding fractions was first introduced by Murphy [11]. A study of their construction and properties has been carried out by McCabe [8] and [9] and by McCabe and Murphy [10].

The particular continued fraction (2.7) converges throughout the finite complex 
plane but unlike the expansion (2.4) the character of its convergence is different in one part of the plane than in the remainder. When $z$ lies outside the region $|\arg z|<\pi / 4$ convergence of (2.7) is faster than that of (2.3), slower than that of (2.4), and like both of these expansions it becomes worse as $|z|$ increases. Inside the region given by $|\arg z|<\pi / 4$ the convergence of (2.7) is good for both small and large values of $|z|$. For the particular continued fraction (2.7) this type of behavior was noticed by Wynn [13] who obtained the same expansion as a special case of a continued fraction expansion of the ratio of two confluent hypergeometric functions. In the same work he also tabulates the numerical efficiency of the three expansions (2.4), (2.5) and (2.7). In [14] the same author obtains converging factors for the expansion (2.7).

3. Truncation Error Estimates. A truncation error estimate for the expansion (2.7) and for (2.4) and (2.6) can be obtained by making use of a fundamental result of Laguerre [5] regarding continued fraction expansions of functions which satisfy first order ordinary differential equations. The result was originally obtained for continued fraction expansions about one point but the extension to expansions about two points is straightforward and is provided by McCabe [8]. Briefly, Laguerre's result is as follows.

The convergents of the continued fractions are rational functions of $z$. The denominator polynomials of these rational functions can be expressed as hypergeometric functions (those of the convergents of (2.4), (2.6) and (2.7) are Laguerre polynomials), and each can thus be regarded as the polynomial solution of a second order ordinary differential equation. Following the work of Laguerre, it can then be shown that a second solution of this equation is in each case a constant multiple of the quantity $\left\{B_{n}(z) F(z)-A_{n}(z)\right\} G(z)$ where $A_{n}(z) / B_{n}(z)$ denotes the $n$th convergent of the continued fraction expansion of $F(z)$ and $G(z)$ is the integrating factor of the first order ordinary differential equation satisfied by $F(z)$. This second solution can also be expressed as a hypergeometric function and hence the truncation error $F(z)-A_{n}(z) / B_{n}(z)$ can be expressed as a ratio of two hypergeometric functions.

Using Laguerre's result the truncation errors of the expansion (2.7) can, after some manipulation, be expressed exactly as

$$
F(z)-\frac{A_{n}(z)}{B_{n}(z)}=\frac{\pi n ! e^{-2 z^{2}} z^{2 n+1}{ }_{1} F_{1}\left(\frac{1}{2}, n+\frac{3}{2} ; z^{2}\right)}{2 \Gamma\left(n+\frac{3}{2}\right) \Gamma\left(n+\frac{1}{2}\right)_{1} F_{1}\left(\frac{1}{2}, \frac{1}{2}-n,-z^{2}\right)} .
$$

Corresponding results for the two expansions (2.4) and (2.6) are given by Luke [7] and by Fair [3], both of whom study the problem of obtaining truncation error estimates of Padé approximations. The convergents of (2.4) and (2.6) are Padé quotients of the respective power series.

Expressing the truncation errors exactly as ratios of hypergeometric functions does not lead directly to useful error estimates. The next step is to make use of known asymptotic estimates of the hypergeometric functions as one or more of the parameters becomes large. For the particular case of (3.1) this is a straightforward procedure (details are given by McCabe [8]), and we obtain the estimate

$$
F(z)-\frac{A_{n}(z)}{B_{n}(z)}=\frac{n ! 2^{2 n} e^{-2 z^{2}} z^{2 n+1}}{(2 n+1)(2 n-1)^{2}(2 n-3)^{2} \cdots 5^{2} 3^{2}} .
$$


For real values of $z$ we can use (3.2) to provide estimates of the errors incurred when (2.7) is used to supply rational approximations to Dawson's integral. For any value of $n$ the above estimate imitates the true error in that it is zero at the origin, tends to zero as $x$ tends to infinity, and reaches a maximum at a finite value of $x$. In the case of the estimate the maximum occurs when $x=\frac{1}{2} \sqrt{2 n+1}$ and experience shows that the position of maximum error of the true errors can be expected to move away from the origin as $n$ increases. The table below shows the position of maximum error of the first ten convergents of (2.7), calculated numerically, and also the position of the maximum estimated error.

\begin{tabular}{ccc}
$n$ & $\frac{1}{2} \sqrt{2 n+1}$ & $\begin{array}{c}\text { maximum } \\
\text { error }\end{array}$ \\
\hline 1 & 0.8660 & 1.0467 \\
2 & 1.1180 & 1.2085 \\
3 & 1.3228 & 1.3665 \\
4 & 1.5000 & 1.5160 \\
5 & 1.6583 & 1.6571 \\
6 & 1.8028 & 1.7903 \\
7 & 1.9364 & 1.9168 \\
8 & 2.0615 & 2.0370 \\
9 & 2.1794 & 2.1520 \\
10 & 2.2913 & 2.2631
\end{tabular}

The error estimate (3.2) was obtained on the assumption that $n$ was large. However, as can be seen from the following table, it will provide reasonably good error estimates even when $n$ is small. On the other hand when $\left|z^{2}\right|$ becomes approximately equal to $n$ the exponential term causes the estimate to tend to zero, as $z$ increases, much faster than the real error. This could be overcome if we could obtain asymptotic estimates of their hypergeometric functions as both $n$ and $z$ become large. In the following table the upper and lower figures are the actual error and estimated error respectively.

\begin{tabular}{|c|c|c|c|c|c|c|}
\hline$x$ & $F(x)$ & $n=2$ & $n=4$ & $n=6$ & $n=8$ & $n=10$ \\
\hline 0.00 & 0.00000000 & - & - & - & - & - \\
\hline 0.25 & 0.23983916 & $\left\{\begin{array}{l}0.60(-3) \\
0.61(-3)\end{array}\right.$ & $\begin{array}{l}0.21(-6) \\
0.21(-6)\end{array}$ & $\begin{array}{c}- \\
0.28(-10)\end{array}$ & $\begin{array}{c}- \\
0.19(-14)\end{array}$ & $\begin{array}{c}- \\
0.85(-20)\end{array}$ \\
\hline 0.50 & 0.42443638 & $\left\{\begin{array}{l}0.13(-1) \\
0.13(-1)\end{array}\right.$ & $\begin{array}{l}0.72(-4) \\
0.72(-4)\end{array}$ & $\begin{array}{l}0.15(-9) \\
0.15(-6)\end{array}$ & $\begin{array}{l}0.17(-9) \\
0.17(-9)\end{array}$ & $\begin{array}{c}- \\
0.12(-12)\end{array}$ \\
\hline
\end{tabular}




\begin{tabular}{|c|c|c|c|c|c|c|}
\hline$x$ & $F(x)$ & $n=2$ & $n=4$ & $n=6$ & $n=8$ & $n=10$ \\
\hline 0.75 & 0.52301277 & $\left\{\begin{array}{l}0.48(-1) \\
0.59(-1)\end{array}\right.$ & $\begin{array}{l}0.14(-2) \\
0.15(-2)\end{array}$ & $\begin{array}{l}0.16(-4) \\
0.16(-4)\end{array}$ & $\begin{array}{l}0.91(-7) \\
0.92(-7)\end{array}$ & $\begin{array}{l}0.33(-9) \\
0.33(-9)\end{array}$ \\
\hline 1.00 & 0.53807951 & $\left\{\begin{array}{l}0.84(-1) \\
0.96(-1)\end{array}\right.$ & $\begin{array}{l}0.76(-2) \\
0.84(-2)\end{array}$ & $\begin{array}{l}0.27(-3) \\
0.28(-3)\end{array}$ & $\begin{array}{l}0.50(-5) \\
0.51(-5)\end{array}$ & $\begin{array}{l}0.57(-7) \\
0.57(-7)\end{array}$ \\
\hline 1.50 & 0.42824907 & $\left\{\begin{array}{l}0.79(-1) \\
0.60(-1)\end{array}\right.$ & $\begin{array}{l}0.22(-1) \\
0.26(-1)\end{array}$ & $\begin{array}{l}0.40(-2) \\
0.45(-2)\end{array}$ & $\begin{array}{l}0.39(-3) \\
0.41(-3)\end{array}$ & $\begin{array}{l}0.23(-4) \\
0.23(-4)\end{array}$ \\
\hline 2.00 & 0.30134039 & $\left\{\begin{array}{l}0.36(-1) \\
0.76(-2)\end{array}\right.$ & $\begin{array}{l}0.13(-1) \\
0.11(-1)\end{array}$ & $\begin{array}{l}0.48(-2) \\
0.57(-2)\end{array}$ & $\begin{array}{l}0.14(-2) \\
0.16(-2)\end{array}$ & $\begin{array}{l}0.27(-3) \\
0.29(-3)\end{array}$ \\
\hline 2.50 & 0.22308372 & $\left\{\begin{array}{l}0.13(-1) \\
0.26(-3)\end{array}\right.$ & $\begin{array}{l}0.35(-2) \\
0.88(-3)\end{array}$ & $\begin{array}{l}0.15(-2) \\
0.12(-2)\end{array}$ & $\begin{array}{l}0.72(-3) \\
0.82(-3)\end{array}$ & $\begin{array}{l}0.29(-3) \\
0.35(-3)\end{array}$ \\
\hline 3.00 & 0.17827103 & $\left\{\begin{array}{l}0.47(-2) \\
0.26(-5)\end{array}\right.$ & $\begin{array}{l}0.75(-3) \\
0.18(-4)\end{array}$ & $\begin{array}{l}0.26(-3) \\
0.50(-4)\end{array}$ & $\begin{array}{l}0.12(-3) \\
0.74(-4)\end{array}$ & $\begin{array}{l}0.68(-4) \\
0.67(-4)\end{array}$ \\
\hline
\end{tabular}

Department of Applied Mathematics University of St. Andrews

St.Andrews, Fife, Scotland

1. W. J. Cody, K. A. Paciorek \& H. C. Thacher, "Chebyshev approximations for Dawson's integral," Math. Comp , v. 24, 1970, pp. 171-178. MR 41 \#2883.

2. H. G. Dawson, “On the numerical value of $\int_{0}^{h} e^{x^{2}} d x$," Proc. London Math. Soc., v. 29, 1898 , pp. $519-522$.

3. W. FaIR, "Padé approximations to the solution of the Ricatti equation," Math. Comp., v. 18, 1964, pp. 627-634. MR 29 \#6630.

4. D. G. Hummer, "Expansion of Dawson's function in a series of Chebyshev polynomials," Math. Comp. , v. 18, 1964, pp. 317-319. MR 29 \#2967.

5. E. LAGUERRE, "Sur le réduction en fractions continues d'une fraction qui satisfait à une équation différentielle linéaire du premier ordre dont les coefficients sont rationnels," J. Math. Pures Appl., v. 1, 1885.

6. B. Lohmander, \& S. Rittsten, "Table of the function $y=e^{-x^{2}} \int_{0}^{x} e^{t^{2}} d t$," Kungl. Fysiogr. Sällsk. $i$ Lund Forh., v. 28, 1958, pp. 45-52, MR 20 \#1427.

7. Y. LuKe, "The Padé table and the $\tau$ method," J. Mathematical Phys. , v. 37, 1958, pp. 110-127. MR 20 \#5558.

8. J. H. MCCABE, Continued Fraction Methods With Applications to First Order Ordinary Differential Equations, Ph D. Thesis, Brunel University, 1971.

9. J. H. McCABE, "An extension of the Padé table to include two point Padé approximations" (To appear.) 
10. J. H. MCCABE \& J. A. MurPhy, "Continued fraction expansions about two points" (To appear.)

11. J. A. Murphy, Rational Approximations to the Error Function, Internal project, Brunel University, 1966.

12. H. C. Thacher, Computation of the Complex Error Function by Continued Fractions, Blanch Anniversary volume, B. Mond (editor), Aerospace Research Laboratories, Washington, D. C.

13. P. WynN, "The numerical efficiency of certain continued fraction expansions. IB," Nederl. Akad. Wetensch. Proc. Ser. A, v. 65 =Indag. Math.,v. 24, 1962, pp. 138-148. MR. 25 \#2690b.

14. P. WynN, “Converging factors for continued fractions. I,II," Numer. Math., v. 1, 1959, pp. 272-320. MR 22 \#6953. 